(C)2009 IEEE. Personal use of this material is permitted. However, permission to reprint/republish this material for advertising or promotional purposes or for creating new collective works for resale or redistribution to servers or lists, or to reuse any copyrighted component of this work in other works must be obtained from the IEEE. 


\title{
Ascertaining the Financial Loss from Non-dependable Events in Business Interactions by using the Monte Carlo method
}

\author{
Omar Hussain and Tharam Dillon \\ Digital Ecosystems and Business Intelligence Institute \\ Curtin University of Technology, Perth Australia \\ O.Hussain@cbs.curtin.edu.au
}

\begin{abstract}
Risk Assessment in business interactions is carried out to determine beforehand the occurrence of undesirable events and their associated consequences. In the literature, approaches have been proposed by which an interaction initiating agent can ascertain the occurrence of undesirable event/s and determine their consequences in an interaction. But those approaches just consider those events that are related to the performance of the other agent, with whom the interaction initiating agent is forming an interaction. It is possible that there may also be such events that are not dependent on the other agent's performance, but will directly or indirectly have an impact on the successful completion of the business interaction. In this paper, we will highlight the importance of considering such event/s during the process of risk assessment, and propose a methodology by which the interaction initiating agent can determine and quantify their effect on the successful completion of its business interaction.
\end{abstract}

Keywords- risk assessment, financial risk, uncertainty, dependable events, non-dependable events.

\section{INTRODUCTION}

Business Interactions are carried out with the aim of achieving certain specific outcomes that are consequential for the progression, advancement and sustenance of user/s involved. It is likely that a user in such interactions will have its financial resources at stake in order to achieve its outcomes. Subsequently, failure to achieve those specific outcomes might have far-reaching consequences for the users involved. In today's competitive business world, the aim of each user is to avoid any such losses and at the same time to achieve maximum returns and benefits. This can be achieved by taking informed decisions in such interactions. By 'informed decision', we mean that decision by which the user's interaction experience and expected benefits are maximized and any losses or undesired outcomes are eliminated or avoided. So the user, before deciding on an interaction in such environments, should consider the concepts that help to make its interaction based decision an informed one.

It is well documented in the literature that 'Risk' is one of the important concepts to consider and analyze when making a decision about any form of interaction [1-4]. Risk is a determinal term which expresses the 'negative' elements that could be possible in an interaction. The user by analyzing the level of risk, can determine the direction in which its interaction might head due to the occurrence of undesired outcomes, or the absence of desired outcomes, as well as the consequences that it could experience as a result of forming an interaction. Such an analysis of risk is an important influence on the behavior of the user in forming a business interaction, as the decision to buy is related to the cost-benefit analysis [5]. Hence, in order for a user to achieve its aims, the manifestation of the level of risk in its interactions is very important. It is by considering this manifestation that a user transforms itself into an intelligent agent that can make decisions according to which it can achieve its aim. Thus, it commands a central role in any discussion related to decision-making. Similarly, decisionmaking in business interactions is no exception to this principal. The occurrence of undesired outcome/s which is captured by risk analysis is due to the uncertainty associated with the interaction. This uncertainty can be captured and managed to a tolerable level by the interacting user through the process of risk management. But for risk management, it is important for the interacting agent to first carry out the process of risk identification and risk assessment as these identify the undesired events and their associated consequences in an interaction. Based on such analysis, steps can be formulated by which the uncertain outcomes can be managed or reduced to a tolerable level in a business interaction.

The specific sub-categories for which risk should be analyzed according to its object of analysis in the context of business interactions for decision making are 'performance risk' and 'financial risk'. By considering performance risk and financial risk as its subcategories the likelihood and magnitude of perceived risk can be determined and considered, as the need to determine these characteristics of risk in business interactions is important. In this paper we will propose a methodology by which one subcategory of risk, the financial risk can be determined before initiating a business interaction. Further in this paper, we will term the interacting agents as the 'risk assessing agent' and 'risk assessed agent'. The risk assessing agent is the initiator of the interaction. In other words, it is that agent that wants to achieve certain desired outcomes and subsequently invests and has financial resources at stake. The agent with which the risk assessing agent interacts is termed the risk assessed agent. In other words, a risk assessed agent is that agent which has the capability to provide the risk assessing agent with its desired outcomes. In the next section, we will identify and categorize the different types of events associated in a business interaction that need to be captured by the interacting agent for risk assessment and management. 


\section{IDENTIFYING THE TYPES OF EVENTS ASSOCIATED WITH RISK IN BUSINESS INTERACTIONS}

We consider that there are three types of outcomes possible as a result of a business interaction. They are:

1. Neutral outcome. We define a neutral outcome as that which relates to the occurrence of those expected events which the interacting user was expecting to achieve as a result of the interaction and from which it will benefit.

2. Positive outcome. We define a positive outcome as that which relates to the occurrence of those unexpected events from which the interacting user will benefit.

3. Negative outcome. We define negative outcome as that which relates to the occurrence of those expected or unexpected events from which the interacting user will experience loss in the interaction.

It is fair to say that a risk assessing agent wants to achieve only a neutral and positive outcome for the successful completion of its business interaction, and avoid the occurrence of a negative outcome as much as possible. This is because a negative outcome signifies the occurrence of those events that will result in loss in the business activity, which are subsequently termed as 'Risk' in the interaction. In contrast to the approaches in the literature which define risk as being associated with unbiased or positive outcomes, we consider that risk which highlights and expresses the level of loss in the business activity is associated only with the occurrence of negative outcomes. So, during the process of risk assessment, it is important to identify any events which could be responsible for the occurrence of a negative outcome in an interaction. This involves dealing with uncertainty.

The uncertainty that has to be determined should be in those events (which we term as expectations) that the risk assessing agent wants to achieve as a result of its business interaction with the risk assessed agent. Expectations are defined as the collection of desired outcomes which the risk assessing agent wants to achieve as a result of its interaction with the other agent. We consider that the risk assessing agent, before initiating the interaction, forms the expectations of its interaction with the risk assessed agent. By doing so, both the interacting agents know how they should act and behave for the successful completion of the interaction. The assessment criteria of the expectations can be broadly divided into two different categories. The first types of events are those for which the risk assessing will depend on the risk assessed agent to achieve. Failure to achieve these outcomes might result in the risk assessing agent experiencing failure in its interaction along with financial consequences. We term such events the 'dependable events' of the interaction. The uncertainty associated with these types of events is the risk assessing agent being unsure whether the other agent will act and behave as promised, based on which it will achieve its desired outcomes. The second category of events from the expectations are those which are outside the scope of dependence on the risk assessed agent, but which will have a direct impact on the successful completion of the risk assessing agent's interaction. We term such events as the 'non-dependable events' of the interaction. The uncertainty associated with them is that the risk assessing agent is unsure of those events occurring as desired or planned in the expectations. The financial loss that could be experienced from such non-dependable events is different from what could be experienced from the dependable events, and hence they too come under the category of undesired outcomes which might have financial consequences as a result of their occurrence. Subsequently, such events too should be identified and assessed during risk analysis in a business interaction. In other words, while determining the financial risk in a business interaction, it is important for the risk assessing agent to take into consideration the financial loss that could be experienced from non-dependable events, apart from also considering the loss due to the dependable events.

In this paper, our aim is to develop a methodology by which the risk assessing agent can determine the financial consequences that it can experience in its business interaction due to the non-dependable events. It can be argued that the uncertainty associated with dependable and non-dependable events in an interaction might not always result in an outcome that has financial loss or financial consequences. It might also result in such outcomes that can be considered as a benefit to the risk assessing agent. But our discussion in this paper is on risk assessment with the aim of determining the possible financial loss in a business interaction. As such, we consider only the occurrences of undesired negative events that will result in financial loss in an interaction. The paper is organized as follows. In Section 3 , we formulate the problem definition. In Section 4, we discuss the related work from the literature according to the defined problem. In Section 5 and Section 6, we propose the methodology for determining the financial loss from nondependable events in the interaction. Finally, in Section 7 we conclude the paper.

\section{PROBLEM DEFINITION}

In order to formulate the problem definition and highlight the importance of considering the non-dependable events while risk analysis, let us consider the following business interaction scenario. Individual ' $A$ ' wants to choose a logistics company with which to interact in order to move its goods from London, England to Perth, Australia. Before doing so, Individual ' $A$ ' wants to analyze the level of risk when deciding upon the logistics company with which it will form an interaction. But before analyzing the level of risk, agent ' $A$ ' should first form the expectations of its business interaction. For explanation sake, let us consider that the assessment criteria formed in the expectations of the risk assessing agent ' $A$ ' interaction with the risk assessed agent are:

- The logistics company should pack the goods properly at the pick up address $(\mathrm{C} 1)$.

- The goods should reach the destination in 5 days (C2).

- The goods should reach the destination undamaged (C3). 
- The logistics company should provide a track and trace facility (C4).

- The total cost for transferring the goods is 6000 Euros. Agent ' $A$ ' should pay half of the amount when the goods are picked up (C5).

- Once the goods are delivered, Agent ' $A$ ' should make the remaining payment of 3000 Euros in equivalent Australian Dollars to the logistics company's branch office at Perth on 15/10/2008 (C6).

In the literature, approaches have been proposed by which one of the subcategory of risk i.e. financial risk that could be experienced in an interaction is determined and quantified [6-8]. But those approaches only consider assessment criteria $\mathrm{C} 1-\mathrm{C} 4$ while determining the possible financial loss. In other words, those approaches consider only those assessment criteria which are dependent on the risk assessed agent's performance, for analyzing the level of financial risk in an interaction. Assessment criteria C6 are not considered by those approaches as they are not related to the performance of the risk assessed agent and hence are not considered as a 'threat' in the interaction. But in reality, assessment criteria C6 too can cause financial loss to the risk assessing agent in an interaction and hence should be considered as 'threat'. These assessment criteria come under the non-dependable events of an interaction. For example, it is possible that the exchange rate between the Australian Dollar against the European Euro might vary after the risk assessing agent ' $A$ ' initiates an interaction with a risk assessed agent. As a result, the amount which the risk assessing agent ' $A$ ' has to pay to the logistics company as mentioned in assessment criteria $\mathrm{C} 6$, might be more than initially anticipated while forming the expectations. The extra investment which has to be made by the risk assessing agent ' $A$ ' in order to complete its expectations, as opposed to what was decided initially, can be considered as being a 'loss' to it. Subsequently, the risk assessing agent while determining the financial risk should also consider this level of loss, apart from the loss that could be experienced from the assessment criteria $(\mathrm{C} 1-\mathrm{C} 4)$ that are dependent on the risk assessed agent.

The above discussion provides a very brief insight into the non-dependable uncertain events and the impact or consequences that they can have on an interaction. The occurrences of such non-dependable events in today's world are quite common when one considers the volatility of the current market situation. For example, in September 2008 the world's markets were in financial turmoil causing billons of dollars to be wiped off from the trading places all around the world. Subsequently, such events directly or indirectly have an effect (positive or negative) on business interactions in different ways. In today's modern world, there might be various such non-dependable events that are important to consider and whose effects must be determined on the successful completion of an interaction. As a result, it is very important for the risk assessing agent to consider such events and factors and to determine their negative impacts while ascertaining the financial loss in business interactions, apart from considering the dependable events.
In the next section, we will discuss the related work from the literature which quantifies and determines the financial consequences from non-dependable events. In Section 5, we will propose a methodology by which such non-dependable uncertain events can be accounted for while determining the financial loss in business interactions.

\section{RELATED WORK}

As mentioned earlier, the specific sub-categories for which risk should be analyzed according to its object of analysis in the context of business interactions for decision making are 'performance risk' and 'financial risk'. By considering performance risk and financial risk as its subcategories, the likelihood and magnitude of perceived risk can be determined and considered, as the need to determine these characteristics of risk in business interactions is important. Approaches have been proposed in the literature which analyzes risk either as a uni subcategory outcome or as a combination of both these subcategories. In this paper, we are interested in studying only those approaches which ascertain the consequences, cost, utility or loss in an interaction as a result of its inherent risk [8-12]. But by doing an analysis of those approaches, we note that they determine the level of financial loss in an interaction because of not achieving the expected desired outcomes from it, due to the failure of the other agent. In other words, they just consider the dependable events and do not take into consideration the existence of non-dependable uncertain events in an interaction and subsequently fail to determine the financial loss from those events. But as mentioned earlier, for risk analysis it is important that the risk assessing agent analyze and consider such nondependable events. In the next sections, we will propose a methodology by which the uncertainty in such events can be considered and their impact is determined on the successful completion of a business interaction.

\section{Determining THE IMPACT OF NON-DEPENDABLE EVENTS ON A BUSINESS INTERACTION}

It is important to note that the non-dependable uncertain events are specific to the business interaction in which they have to be determined. In other words, such events vary according to the different outcomes that the risk assessing agent wants as a result of its business interaction. Subsequently, in order for the risk assessing agent to determine the impact of such events on the successful completion of its business activity, it should first identity them according to the specific interaction in question. Further in the paper, we term the non-dependable events (NDE) in the interaction as:

$$
\left\{\mathrm{NDE}_{1}, \mathrm{NDE}_{2}, \mathrm{NDE}_{3}, \ldots \ldots \ldots, \mathrm{NDE}_{\mathrm{n}}\right\}
$$

where: ' $n$ ' represents the number of non-dependable events identified by the risk assessing agent in a business interaction. 
Once the non-dependable events of an interaction have been identified, the risk assessing agent should then determine the possible scenarios by which they will have an impact on the successful completion of its interaction. By 'impact' we mean the financial loss or financial consequences that they might present to the risk assessing agent in the interaction. This involves the risk assessing agent dealing with uncertainty associated with those events and determining their level of occurrence, which will result in experiencing impact in the interaction. But unlike dependable events, the occurrence of non-dependable events might be spontaneous and volatile that it is extremely difficult for the risk assessing agent to have a distribution of any sort which models the trend or chances of their occurrence during the interaction time period. But on the other hand, it is also important for the risk assessing agent to account for the uncertainty of such factors and determine the expected financial loss from them in order to have a successful business interaction.

So we propose that the risk assessing agent, in order to model the uncertainty associated with the non-dependable events, utilize the Monte Carlo technique in preference to probability distribution methods. The Monte Carlo technique is a sampling method which uses simulations of independent random numbers to model the uncertainty associated with the problem. It is a method for analyzing uncertainty propagation, with the goal of determining their effect on the output of the system. It is not a model which gives a deterministic output at each simulation, but is a method for iteratively evaluating a deterministic model (usually the problem framework) using sets of random variables as inputs [13]. The Monte Carlo simulation is best utilized when the underlying model is complex, nonlinear and involves uncertain parameters. Hence, it is best suited to our problem of determining the expected loss in business interactions due to non-dependable, uncertain events. Another reason for utilizing the Monte Carlo technique in our approach instead of probability modeling techniques is to avoid the disadvantage of probability distributions while modeling future value/s of a variable over a given period of time. In probability distributions, the non-zero value of occurrence must be assigned to an element from the UoD of the particular uncertain variable, whose likelihood of occurrence is very high. Furthermore, whatever probability is assigned to that element from the UoD of the variable, affects the probability value to be assigned to the other elements of that variable, as the sum of the probability values of all the elements from the UoD of a variable should be equal to 1 . Subsequently, the uncertainty associated with that variable at a future point in time cannot be represented and modeled accurately by using probability distributions.

The Monte Carlo technique requires the risk assessing agent to specify a range in which each uncertain input variable/s might fluctuate during the time period of the interaction. The range specifies the lower and upper bounds, between which that variable can take a value in the time period of the interaction. The risk assessing agent can do it by observing the trend of that uncertain variable over a given past period of time. When the simulations are run, the
Monte Carlo technique for each input variable generates a random value between its bounds with a probability of occurrence of 1 , and then ascertains its impact on the output function. If there are more than one non-dependable variable in the problem definition, then a random value is generated for each of them within their bounds for each simulation, and their corresponding effect on the output function is determined. The value generated in each simulation will be according to a certain value of confidence interval, which has to be specified by the risk assessing agent. In our model, we consider the confidence interval value to be $99 \%$ during the simulations.

In order to explain with an example, let us consider the business interaction scenario discussed in Section 3. Let us assume that the risk assessing agent ' $A$ ' wants to interact with a logistics company during the time period 01/10/2008 - 15/10/2008. As discussed earlier, from the formed expectations, assessment criteria $\mathrm{C} 5$ and $\mathrm{C} 6$ are not dependent on the risk assessed agent's performance and are the non-dependable events of the interaction. The risk assessing agent does not expect to experience any financial loss from assessment criteria $\mathrm{C} 5$, as it will be completed at the time spot (start time) of the interaction; whereas, assessment criteria C6 is scheduled to be completed at the end of the business interaction. Let us consider that agent ' $A$ ' formed the expectations on 30/9/2008 by considering that 1 Australian Dollar (AUD) converts to 0.572 Euros (EUR). So according to the expectations, the risk assessing agent has to pay a financial amount of 5245 in Australian dollars (which is equivalent to 3000 Euros) to the risk assessed agent on $15 / 10 / 2008$, in order to successfully complete its business activity. But by considering the recent financial situation in the world's market, it is possible that the exchange rate may vary till the time of completion of its interaction, resulting in the risk assessing agent having to pay a financial amount that is different from the one decided in the expectations. The resulting financial amount to be paid might be the same, or might vary from what was decided in the expectations. If it is the same amount or even less than what was decided in the expectations, then the risk assessing agent will not experience any financial loss from this non-dependable assessment criterion. On the other hand, if the resulting amount which the risk assessing has to pay is more than what was decided in the expectations, then subsequently this criterion will result in the risk assessing agent experiencing financial loss in the business interaction. So in order to make an informed interaction-based decision, it is important that agent ' $A$ ' ascertains the financial loss that it can experience due the assessment criterion $\mathrm{C} 6$, apart from just considering the other assessment criteria (C1-C4) during risk analysis.

Let us consider that agent ' $\mathrm{A}$ ', by considering the market volatility and the past conversion trends, determines the range of the input variable as $\{0.51,0.66\}$. Utilizing MATLAB to run the Monte Carlo simulations on this range with 5000 iterations and with $99 \%$ confidence level, Agent ' $A$ ' will have the different output values that represent the impact of the non-dependable variable on its interaction as shown in Figure 1, due to its occurrence in a way other than 
what was initially expected. In other words, the values obtained from Monte Carlo simulation represents the different levels of financial amount that the risk assessing agent might have to pay to the risk assessed agent, depending upon the various possible permutations of the nondependable variable in its range. It should be noted that Figure 1 represents a part of the results obtained from the Monte Carlo simulation, for the current business example with 5000 simulations. Based on the simulation values, the risk assessing agent should determine the level of financial loss that it can experience in the interaction, due to the variation in the occurrence of the non-dependable event; from what was initially expected. In the next section, we will propose a methodology by which the risk assessing agent can determine the level of financial loss to it due to the nondependable event, from the simulation results.

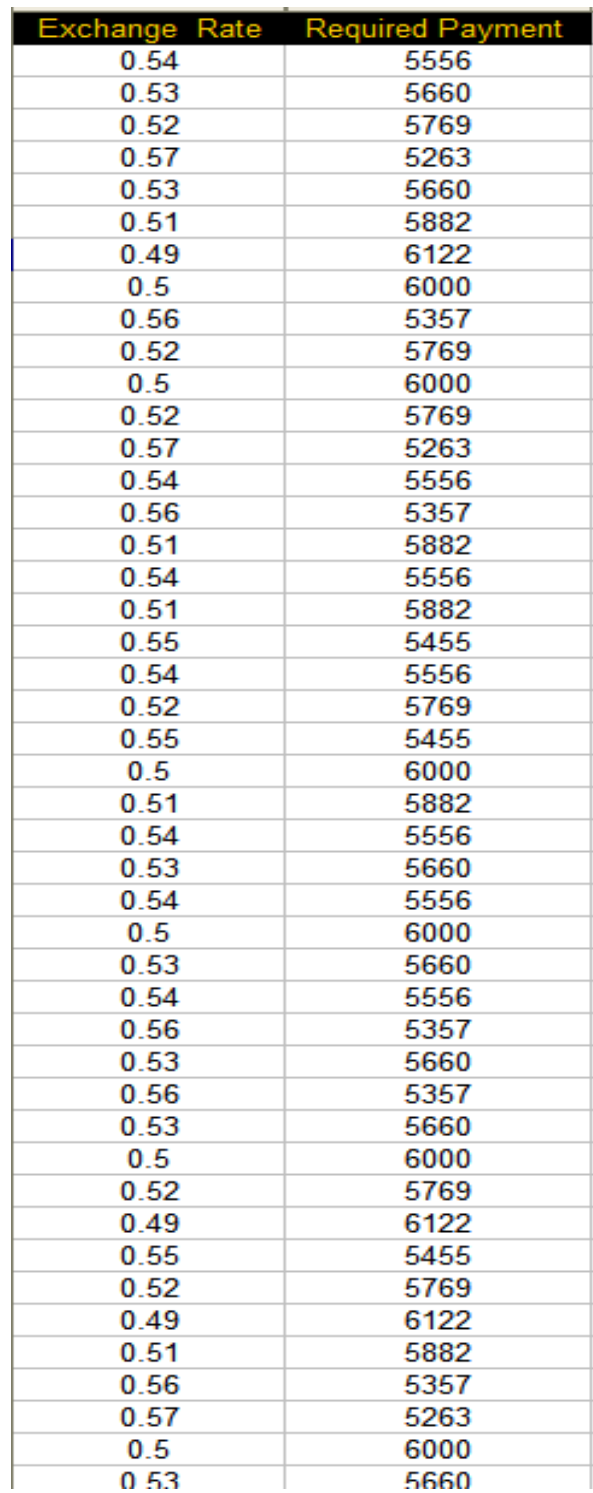

Figure 1. Monte Carlo simulations output based on the different permutations of the non-dependable variable

\section{DETERMINING THE FINANCIAL LosS IN A BUSINESS INTERACTION DUE TO NON-DEPENDABLE EVENTS}

Once the simulation with the desired number of iterations is complete, the risk assessing agent will have the different financial amounts that it might have to pay to the risk assessed agent depending upon the various permutations of the uncertain input variable. To assimilate the results and to determine the possible loss that it could experience, we propose that the risk assessing agent, from the simulation results should plot the Factual Cost Curve (FCC) of the interaction. Factual Cost Curve as shown in Figure 2 represents the probability of the different amounts which the risk assessing agent might have to pay to the risk assessed agent, due to the different possibilities of occurrence of the non-dependable uncertain variable in its interaction. The Factual Cost Curve (FCC) is plotted by determining the cumulative probability of the risk assessing agent having to pay at least an amount to the risk assessed agent, from the iteration results of the simulation; in order to commit to the assessment criterion.

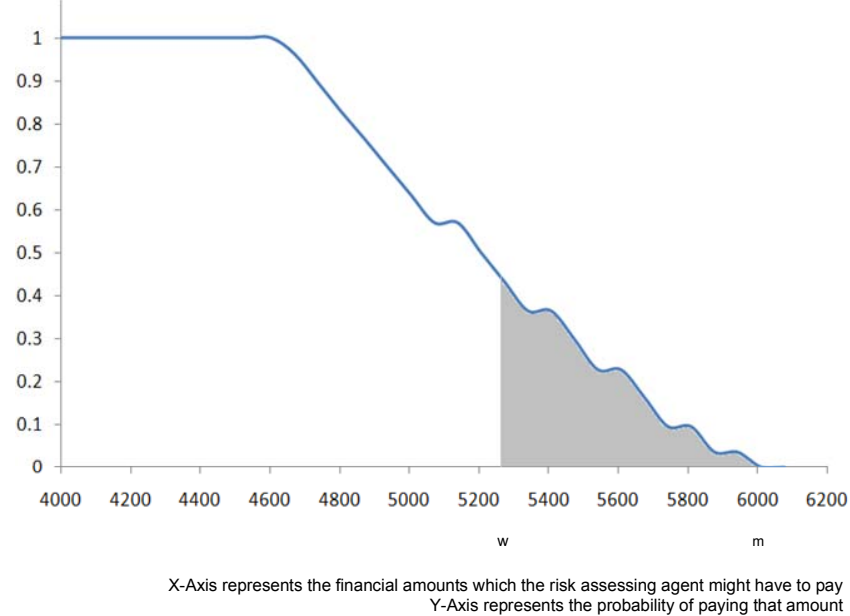

Figure 2. The Factual Cost Curve (FFC) of the Interaction

Continuing the example from the previous section, according to the expectations the risk assessing agent ' $A$ ' had to pay 5245 AUD to the logistic company upon receiving the goods. But due to the uncertainty in the nondependable event, it is possible that agent ' $A$ ' might have to pay the different levels of financial amounts as shown in Figure 2. The extra additional level/s of financial amounts which agent ' $A$ ' might have to pay to the risk assessed agent from what was decided in the expectations, can be considered as the possible loss that could be incurred by it in the interaction from the non-dependable events. To determine the possible expected loss due to the nondependable event, we propose that the risk assessing agent ' $A$ ' should plot its maximum investment capacity (MIC) on the FCC. The maximum investment capacity of the risk assessing agent represents the financial amount that it had to pay, as decided in the expectations of its interaction. So any level of required investments after the risk assessing agent's MIC on the FCC (represented by the shaded part of Figure 2) represents the possible loss it could experience in the 
interaction. This is because that part of the curve represents those additional levels of financial resources with their probability that the risk assessing agent might have to pay in order to adhere to the expectations of the interaction, but theoretically those resources are beyond what was decided initially in the expectations. Hence, those extra required level/s of resources can be termed as the possible loss that the risk assessing agent could experience in the interaction. The risk assessing agent can determine the possible financial loss from uncertain non-dependable events (Financial Loss NDE) by:

$$
\text { Financial Loss }{ }_{\mathrm{NDE}}=\int_{w}^{m} F C C(x)
$$

where: 'w' represents the maximum investment capacity of the risk assessing agent,

' $m$ ' represents the point on the abscissa where the Factual Cost Curve (FCC) ends.

The above equation gives the area under the Factual Cost Curve (FCC) from the point of maximum investment capacity of the risk assessing agent. The degree of financial loss to the risk assessing agent from non-dependable events in an interaction will be proportional to the area of the Factual Cost Curve (FCC), after the maximum investment capacity of the risk assessing agent (point ' $w$ ' in Figure 2). The greater the area of the FCC after point ' $w$ ', the higher will be the degree of financial loss in the interaction and vice versa. By utilizing the proposed methodology, the risk assessing agent can determine the different levels of financial loss that it could experience from non-dependable uncertain events in an interaction, and utilize it while performing risk analysis in an interaction. The example that we considered in this paper is a basic scenario of a business interaction. But the proposed methodology can be applied to any scenarios of a business interaction that deals with the non-dependable events in it. For carrying out a comprehensive analysis of risk, it is imperative for the risk assessing agent to consider and analyze such events apart from those for which it will depend on the risk assessed agent.

\section{CONCLUSION}

In this paper we proposed an approach by which the risk assessing agent in the domain of business interactions can determine the level of financial loss that it can experience due to the non-dependable uncertain events. We utilized the Monte Carlo simulation to model the uncertainty of such events and determine their impact on the successful output in the interaction. It should be noted that the financial loss determined by utilizing this approach is limited only to the non-dependable uncertain events in an interaction. In order to determine the total financial loss in an interaction, the risk assessing agent should combine this level of loss with the financial loss that could be experienced from dependable events in the interaction. This is our future work.

\section{REFERENCES}

[1] Aaron M. Hoffman, "A Conceptualization of Trust in International Relations," European Journal of International Relations, vol. 8, pp. 375-401, 2002.

[2] Bo-chiuan Su, "Risk behavior of Internet shopping: Comparison of College Students' versus Non-Student Adults'," in Proceedings of the 5th international conference on Electronic Commerce (ICEC 2003), Pittsburgh, Pennsylvania 2003, pp. $181-185$

[3] H. R. Rao, "Study Shows Perceived Risk of Online Credit Purchases Linked to Trust, Familiarity with Intermediaries," in University of Buffalo, News Centre, 2007.

[4] Stefano Grazioli and S. L. Jarvenpaa, "Perils of Internet Fraud: An Empirical Investigation of Deception and Trust with Experienced Internet Consumers," IEEE Transactions on Systems, Man and Cybernetics, vol. 30, pp. 395-410, July 2000.

[5] Sander Greenland, "Bounding Analysis as an Inadequately Specified Methodology," Risk Analysis, vol. 24, pp. 10711083, October 2004.

[6] Audun Jøsang and S. L. Presti, "Analysing the Relationship between Risk and Trust," in Second International Conference on Trust Management (iTrust 2004). vol. LNCS 2995 Oxford, United Kingdom: Springer 2004, pp. 135-145.

[7] Colin English, Sotirios Terzis, and W. Wagealla, "Engineering Trust Based Collaborations in a Global Computing Environment," in Second International Conference on Trust Management (iTrust'04). vol. LNCS 2995 Oxford, UK: Springer, 2004, pp. 120-134.

[8] Omar K. Hussain, Elizabeth Chang, Farookh K. Hussain, and Tharam S. Dillon, "Towards Quantifying the Possible Risk in e-commerce Interactions for RDSS," in Proceedings of the IEEE International Conference on e-Business Engineering (ICEBE'07), IEEE, Ed. Hong Kong, China: IEEE, 2007, pp. 89-96.

[9] Colin English, Waleed Wagealla, Paddy Nixon, Sotirios Terzis, Helen Lowe, and A. McGettrick, "Trusting Collaboration in Global Computing Systems " in The First International Conference on Trust Management (iTrust'03), Heraklion, Crete, Greece, 2003, pp. 136-149.

[10] Iris Bohnet and Richard Zeckhauser, "Trust, risk and betrayal," Journal of Economic Behavior \& Organization, vol. 55, pp. 467-484, 2004.

[11] Ching Lin and V. Varadharajan, "Trust Based Risk Management for Distributed System Security - A New Approach," in The First International Conference on Availability, Reliability and Security (ARES'06) Vienna: IEEE Computer Society, 2006, pp. 6-13.

[12] Yuk-Hei Lam, Zili Zhang, and K.-L. Ong, "Trading in Open Marketplace Using Trust and Risk," in Proceedings of the IEEE/WIC/ACM International Conference on Intelligent Agent Technology (WI-IAT'05), Compiegne, France, 2005, pp. 471-474.

[13] James R Evans and David L Olson, Statistics, Data Analysis, and Decision Modeling, 2 ed.: Prentice Hall, 2002.

[14] Didier Dubious, Henri Prade, Henri Farreny, Roger MartinClouaire, Claudette Testemale, and E. F. Harding, Possibility Theory: An Approach to Computerized Processing of Uncertainty. New York: Plenium Press, 1988. 